\title{
The Ophthalmology Development of Hyperbaric Medicine for Treating Central Retinal Artery Occlusion in Hong Kong
}

\author{
Sunny Chi Lik Au ${ }^{*}$, Callie Ka Li Ko ${ }^{1}$ \\ ${ }^{1}$ Department of Ophthalmology, Pamela Youde Nethersole Eastern Hospital, Tung Wah Eastern Hospital, Hong \\ Kong \\ *Corresponding Author: Sunny Chi Lik Au, Department of Ophthalmology, Pamela Youde Nethersole Eastern \\ Hospital, Tung Wah Eastern Hospital, Hong Kong; Email: kilihcua@ gmail.com
}

Received Date: 01-07-2021; Accepted Date: 14-07-2021; Published Date: 22-07-2021

Copyright $^{\odot} 2021$ by Au SCL, et al. All rights reserved. This is an open access article distributed under the terms of the Creative Commons Attribution License, which permits unrestricted use, distribution, and reproduction in any medium, provided the original author and source are credited.

\section{Editorial}

Hong Kong first established the Hyperbaric Oxygen Therapy (HBOT) for treatment of Central Retinal Artery Occlusion (CRAO) since November 2018 [1]. Before the era of hospital-based HBOT in 2018, similar service was provided by the Fire Services Department of the government on a remote island situated Recompression Treatment Center, which was designed to train and treat divers [2]. CRAO was not on the treatment indication list during that diversoriented era. From September 2018 onwards, HBOT public hospital service was provided under the Accident and Emergency department in Pamela Youde Nethersole Eastern Hospital, the only territory public hospital with HBOT chambers currently [3]. CRAO was then included as one of the treatment indications supported by the Undersea and Hyperbaric Medical Society (UHMS) and United States Food and Drug Administration approval [4].

\section{Keywords}

Central Retinal Artery Occlusion; Hyperbaric Oxygen Therapy; Ophthalmology; Hong Kong; HORA Study 


\section{Background}

Few life threatening or organ saving diseases are covered by the public HBOT service in Hong Kong. Other than severe decompression sickness, cerebral arterial gas embolism, carbon monoxide poisoning and necrotizing soft tissue infection, CRAO is the only ophthalmology entity covered by the current public HBOT service [1,2,5,7]. This HBOT referral service is opened to both public and private medical practitioners 24 hours every day [1]. Our service was uninterrupted despite the few waves of novel coronavirus disease 2019 outbreak locally [8-10]. Being the first regional HBOT center for CRAO treatment, our public territory-wide service was also conducted under research basis, the HORA (Hyperbaric Oxygen therapy for central Retinal Artery occlusion) study [1,9-11].

\section{Patient Preparation}

First, do no harm [12]. This is an important treatment principle for treating every patient with HBOT. Past medical history is taken to alert HBOT providers. Particularly, diabetes mellitus, epilepsy, chronic obstructive airway disease, asthma, psychiatric illness, pregnancy will be asked. Besides, pacemaker or implantable cardioverter defibrillator implantation history and pneumothorax history are also essential [13]. Visual Acuity (VA) will be documented before commencement of HBOT to monitor progress, and determine the length of treatment.

\section{Contraindication of HBOT}

A check list of absolute contraindications is established [14]. They include unresolved severe bronchospasm or pneumothorax, untreated tension pneumothorax, pneumocephalus, presence of intraocular gas, hollow orbital prosthesis (Medpor implant is not considered as hollow orbital prosthesis), and currently taking bleomycin or adriamycin. Chest X-ray (CXR) and Electrocardiogram (ECG) will be done before entrance of the HBOT multi-place chamber for screening purpose. Ophthalmological exclusion of cases include those filler injection related, giant cell arteritis and ophthalmic artery occlusion [15-17]. Because choroidal circulations are compromised in these etiologies, thus HBOT will not be effective.

\section{Possible Complications of HBOT}

These can be classified into ocular and systemic complications. For ocular complications, they include visual field narrowing, eyelid twitching, myopia progression and cataract formation [18]. The commonest systemic complication is barotrauma over the middle ear [19]. Other systemic complications are sinus pain, pulmonary oxygen toxicity presenting with squeezing

Au SCL | Volume 2; Issue 2 (2021) | JOAR-2(2)-018 | Editorial

Citation: Au SCL, et al. The Ophthalmology Development of Hyperbaric Medicine for Treating Central

Retinal Artery Occlusion in Hong Kong. J Ophthalmol Adv Res. 2021;2(2):1-8.

DOI: http://dx.doi.org/10.46889/JOAR.2021.2206 
sensation in chest, retrosternal burning sensation, or reversible decline of pulmonary function. Occasionally, central nervous system oxygen toxicity can occur [20].

\section{Treatment Protocol for CRAO}

There are 2 available protocols for treating CRAO in our center, they are referenced to UHMS Hyperbaric Therapy Indications, and the United States Navy Diving Manual. Stepwise decompression takes slightly longer treatment time than the usual one (Fig. 1), but is less commonly used in our practice (Fig. 2).

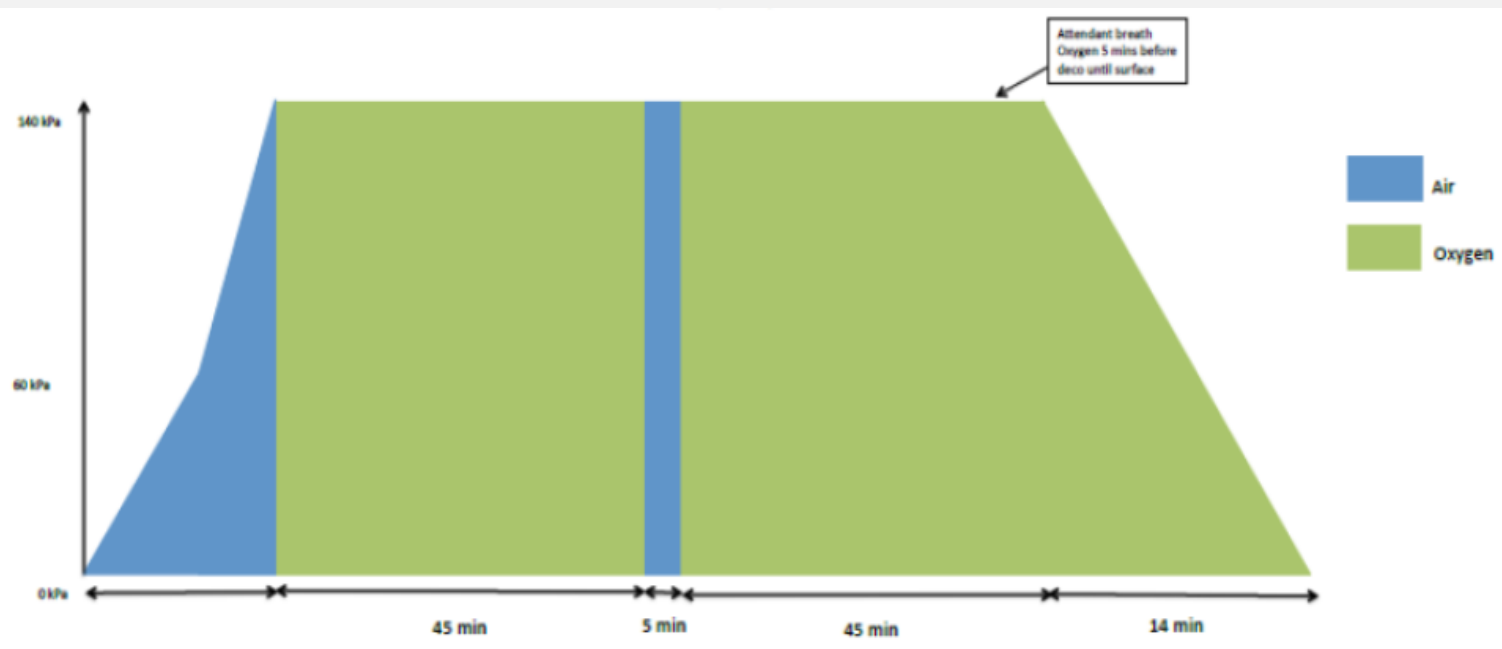

Figure 1: The shorter duration, and less manipulator dependent protocol for treating CRAO.

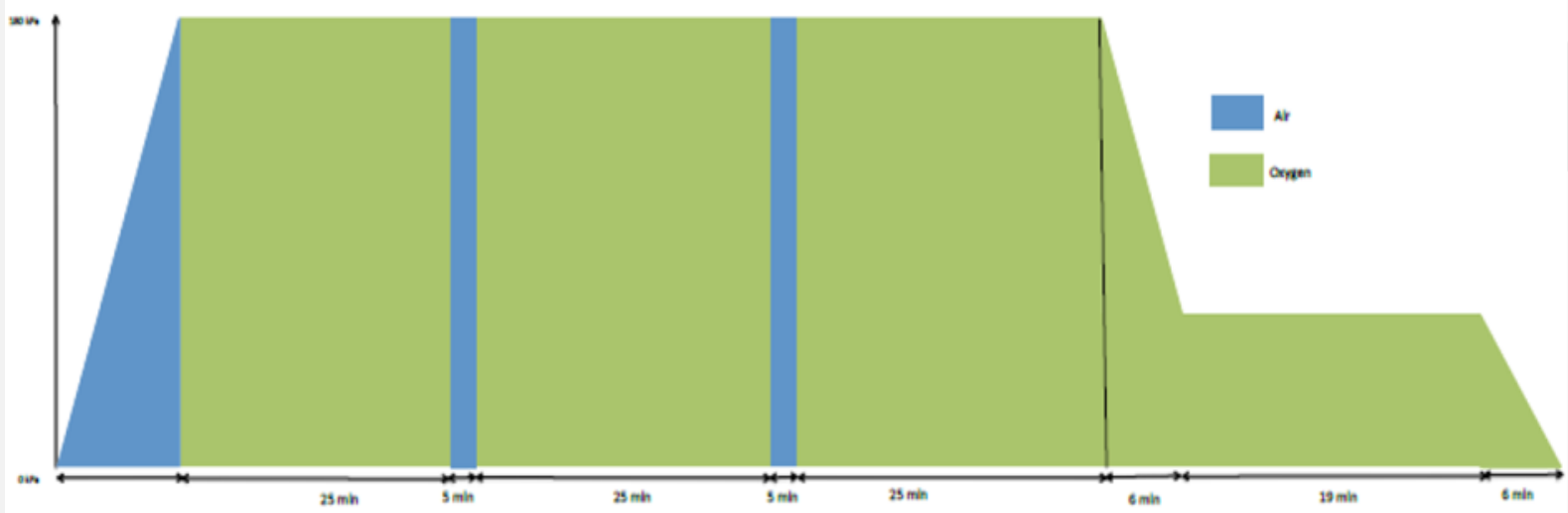

Figure 2: The longer duration, and more complex protocol. 


\section{Practical Operation Manual}

The HBOT center assessment outline (illustrated with a case example)

$<$ HBOT treatment notes $>$

Diagnosis: Right CRAO

New Case

History of Present Illness (HPI):

- Date and Time of symptom onset: 20/11/2019 8 am

- Seen by Ophthalmology at 10:40 am

- VA on presentation: hand movement

Referral Source: Hospital an ophthalmology

Treatment initiated by Eye: Timolol $0.5 \%$

Background

Past Medical History (PMH): Hypertension; eczema; Non-smoker

Diabetes Mellitus: No

Past Eye History: nil

Mobility: Walk with stick

\section{Planning Treatment}

Any difficulty equalizing in the past: no

No Contraindications

Physical exam:

- Vitals stable

- Bilateral Tympanic Membrane (TM) normal

- Chest exam air entry good

- VA before HBOT: Right (R): Finger Counting (FC) at face and Left (L): 0.3 PH 0.4-1

Au SCL | Volume 2; Issue 2 (2021) | JOAR-2(2)-018 | Editorial

Citation: Au SCL, et al. The Ophthalmology Development of Hyperbaric Medicine for Treating Central

Retinal Artery Occlusion in Hong Kong. J Ophthalmol Adv Res. 2021;2(2):1-8. 
CXR: clear

ECG: SR, RBBB

Benefits and risks explained

Consent signed

Precautions: nil

\section{Current Treatment}

Treatment Session Number: \#1

Date and time of treatment: 20/11/2019 16:12 pm

- For $1^{\text {st }}$ Session: Duration from symptom onset to HBOT: 8 hours

Maximum pressure (in ATA): 2.8

Duration of Treatment (in minutes): 125

- HBOT started at 14:51. Failed equalization with bilateral ear blockage sensation and left ear pain, treatment aborted at $106 \mathrm{kPa}$

- R TM mild erythema, L TM grade 1-2 barotrauma

- Bilateral myringotomy performed

- HBOT restarted at 16:12, uneventful after myringotomy

Post-treatment TM: bilateral erythema L>R

Post treatment VA: R eye FC at 1 feet

Dispatch

- Back to ophthalmology ward

- Next treatment 21/11/2019 09:30 


\section{The Ophthalmology Clinic Assessment Outline (Illustrated with the same case example)}

No Known Drug Allergy

PMH: Hypertension, eczema

Past Ophthalmic History: nil

R CRAO

HPI:

- Source of referral: Accidental \& Emergency department of Hospital A

- Date and time of symptom onset: 20/11/2019 8 am

- Symptom to Door Time (Hr): $2.5 \mathrm{hr}$

- Presenting VA (at parent team): FC

- Treatment by parent team: nil

- Appointment at medical: in-patient consult pending

HBOT treatments (date, time, HBOT pressure, complication)

1. $20 / 11 / 19 \mathrm{pm}$

2. $21 / 11 / 19 \mathrm{am}$

\section{Ophthalmology Assessment}

Subjective improve with brighter feeling.

$\mathrm{R}$ diffuse retinal edema, cherry red spot, $\mathrm{L}$ macula and vessels normal.

Fundus Fluorescein Angiography: R mild delayed retinal perfusion to 1:03. L fully perfused by $0: 49$.

Optical Coherence Tomography: R macular edema sparing fovea/ retina swelling+, Central Macular Thickness (CMT) 383. L normal, CMT 242.

Pre- treatment VA (with/ without glasses, + Pinhole (PH)):

Day $1(\mathrm{dd} / \mathrm{mm} / \mathrm{yy}):$ am ----

$$
\text { pm FC without glasses }
$$

Day 2 (dd/mm/yy): am FC without glasses 
pm

Day $3(\mathrm{dd} / \mathrm{mm} / \mathrm{yy})$ : am

pm

Day 4 (dd/mm/yy): am

$\mathrm{pm} *$

*VA after 6 th $/ 7^{\text {th }}$ HBOT treatment* (with/ without glasses, + ph):

Day $5(\mathrm{dd} / \mathrm{mm} / \mathrm{yy}):$ am

$\mathrm{pm}$

Plan:

Proceed to further HBOT: (Yes/No)

Next Eye Follow Up date:

Referral to Medical:

Referral to Parent Team:

Bloods (if any): erythrocyte sedimentation rate

\section{Practical Tips and Future}

Some vitreoretinal surgeons would arrange pars plana vitrectomy with intra-arterial tissue plasminogen activator injection within the same session with the recent advances of such [21]. Intravitreal gas fluid exchange for tamponade is not suggested, if these CRAO patients are subsequently planned for HBOT, as intraocular gas will expand and shrink across HBOT pressure variation. HORA study has now published its third reports [1,9,10]. With the promising VA outcome of HBOT for CRAO and to cope for an increasing demand, a future HBOT center will be available in the coming decades in Hong Kong [1]. We, ophthalmologists, are looking forward to further collaboration with other hyperbaric medicine practitioners to save vision for these ocular stroke patients [22,23].

\section{References}

1. Yip LT, Au SCL, Ko CKL. Hyperbaric oxygen therapy for central retinal artery occlusion: experience in Hong Kong. Hong Kong J Ophthalmol. 2020;24(2):44-50. 
2. Ramaswami RA, Lo WK. Use of hyperbaric oxygen therapy in Hong Kong. Hong Kong Med J. 2000;6(1):108-12.

3. Leung JK, Lam RP. Hyperbaric oxygen therapy: its use in medical emergencies and its development in Hong Kong. Hong Kong Med J. 2018;24(2):191-9.

4. Murphy-Lavoie H, Butler F, Hagan C. Central retinal artery occlusion treated with oxygen: A literature review and treatment algorithm. Undersea Hyperb Med. 2012;39:943-53.

5. Sen S, Sen S. Therapeutic effects of hyperbaric oxygen: integrated review. Med Gas Res. 2021;11(1):30-3.

6. Kirby JP. Hyperbaric oxygen therapy emergencies. Mo Med. 2019;116(3):180-3.

7. Au SCL, Ko STC. An old lady with acute headache and sudden blindness. Hong Kong J Emerg Med. 2021;28(2):121-3.

8. Sunny CLA. Performing hyperbaric oxygen therapy for central retinal artery occlusion under COVID-19: From myringotomy to rapid viral test. Health Policy Technol. 2021;10(1):29-30.

9. Au SCL, Ko CKL. Impact of COVID-19 on acute central retinal artery occlusion patient attendance in Hong Kong: The HORA study brief report number 2. Acta Sci Clin Case Rep. 2021;2:1-2.

10. Au SCL, Ko CKL. Prevalence of SARS-CoV-2 among central retinal artery occlusion patients: A case seriesHORA study report No. 3. J Acute Dis. 2021.

11. Au SCL, Ko CKL. Comments on coronavirus positive patients presenting with stroke-like symptoms. J Stroke Cerebrovasc Dis. 2021;30(7):105741.

12. Hughes G. First do no harm; then try to prevent it. Emerg Med J. 2007;24(5):314.

13. Kot J. Medical devices and procedures in the hyperbaric chamber. Diving Hyperb Med. 2014;44(4):223-7.

14. Gawdi R, Cooper JS. Hyperbaric contraindications. StatPearls. 2021.

15. Au SCL, Ko STC. Giant Cell Arteritis. Eur Arch Med Res. 2020;36(2):159-61.

16. Au SCL, Ko STC. Vision loss due to ophthalmic artery occlusion secondary to spontaneous internal carotid artery dissection. Hong Kong Med J. 2020;26(4):348-9.

17. Au SCL, Ko CKL. In reply: thrombotic neurovascular disease in COVID-19 patients. Neurosurg. 2021;88(6):E573-4.

18. Gengel KC, Hendriksen S, Cooper JS. Hyperbaric related myopia and cataract formation. StatPearls. 2021.

19. Heyboer M, Wojcik SM, Grant WD, Chambers P, Jennings S, Adcock P. Middle ear barotrauma in hyperbaric oxygen therapy. Undersea Hyperb Med. 2014;41(5):393-7.

20. Heyboer M, Sharma D, Santiago W, McCulloch N. Hyperbaric oxygen therapy: side effects defined and quantified. Adv Wound Care (New Rochelle). 2017;6(6):210-24.

21. Sobol EK, Sakai Y, Wheelwright D, Wilkins CS, Norchi A, Fara MG, et al. Intra-arterial tissue plasminogen activator for central retinal artery Occlusion. Clin Ophthalmol. 2021;15:601-8.

22. Au SCL. Acute blindness by central retinal artery occlusion. Vis J Emerg Med. 2020;21.

23. Au SCL, Ko CKL. Ocular stroke and COVID-19. Hong Kong Med J. 2021;27(3):231.

Au SCL | Volume 2; Issue 2 (2021) | JOAR-2(2)-018 | Editorial 